\title{
Genetic Clustering for Polycystic Ovary Syndrome Detection in Women of Reproductive Age
}

\author{
Anuradha D. Thakare, Priyanka R. Lele
}

\begin{abstract}
Now a days, hormonal disorder causing Polycystic Ovary Syndrome (PCOS) is been observed in most of the women of reproductive age. PCOS causes enlarged ovaries with small cysts on the outer edges. Women with PCOS may have irregularity in menstrual periods or excess male hormone (androgen) levels. The ovaries may develop numerous small collections of follicles (cysts) and fail to regularly release eggs. Symptoms of PCOS include irregular periods, excess androgen, polycystic ovaries, abnormal Body Mass Index, disturbed levels of hormones (Luteinizing Hormone, Follicle-stimulating Hormone, Dehydroepiandrosterone), poor insulin resistance.

There is a need to design and develop an optimized system to analyze the sonogram in correlation with the physical symptoms for detection of PCOS at early stage which may result in proper treatment and reduced health loss. This article presents work-in-progress of our proposed research on Intelligent System to detect PCOS. The performance analysis of various Machine learning algorithms like Artificial Neural Network, K- nearest Neighbor and Linear Regression to detect PCOS is presented. Whereas, optimized Genetic Clustering for optimization of classification results is proposed. Basic Genetic Algorithm (GA) and other hybrid GA's will be used for comparing the optimal results. The classification results are optimized with $89 \%$ accuracy
\end{abstract}

Keywords: Classification, Genetic Clustering, Machine Learning, Polycystic Ovary Syndrome, statistical measures, Sonography.

\section{INTRODUCTION}

Disorders of the woman reproductive system can occur as a result of disorder in one of the many various reproductive organs: the ovaries, uterus, cervix, the vagina, or the cause behind occurring of these diseases is hormonal changes inside the body, hormonal imbalance, stress, irregular living patterns, etc. Polycystic ovary syndrome (PCOS) is the most common endocrine disease in women at reproductive age group. It is a condition that disturbs a woman's hormone levels. Women with PCOS produce an high amount of

Revised Manuscript Received on February 05, 2020.

* Correspondence Author

Anuradha D. Thakare*, Department of Computer Engineering, Pimpri Chinchwad College of Engineering, Pune, India.

E-mail: anuradha.thakare@pccoepune.org

Priyanka R. Lele, Department of Computer Engineering, Pimpri Chinchwad College of Engineering, Pune, India.

E-mail: priyankalele7@gmail.com

(C) The Authors. Published by Blue Eyes Intelligence Engineering and Sciences Publication (BEIESP). This is an open access article under the CC BY-NC-ND license (http://creativecommons.org/licenses/by-nc-nd/4.0/) androgen than normal [1]. This hormone imbalance causes abnormality in menstrual cycle and leads to infertility. PCOS also causes hirsutism (unnecessary hair growth on the face and body), and baldness. Polycystic Ovary Syndrome can cause long-time health issues like diabetes and heart sickness. PCOS is a trouble that affects ladies throughout their childbearing years (15 to 44 age)[2]. 5\% - 10\% of women among 15 and 44, or at some point of the years of childbearing, have PCOS [3]. Most women find out they've PCOS of their 20s and 30s, once they have problems getting pregnant and consult their doctor. But PCOS can manifest at any age after puberty. PCOS affects a woman's ovaries with the production of estrogen and progesterone (hormones that regulate the menstrual cycle). Birth manipulates pills and diabetes capsules can assist restoration the hormone imbalance and improve signs. Fig. 1 indicates the cysts formation in the ovary of girls having PCOS.

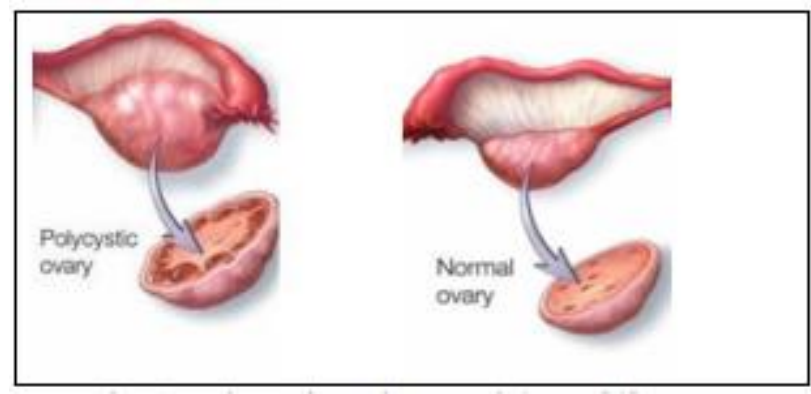

Fig. 1 Polycystic and Normal Ovary[4]

The most common PCOS symptoms are; Irregular durations, Age, BMI, Hirsutism, Acne, Hormone exams and Insulin resistance [5]. The loss of ovulation, the uterus lining does not shed every month due to irregular intervals in the reproductive age. Generally, PCOS may additionally occur after the puberty age (15 to $35 \mathrm{yrs)} \mathrm{.Up} \mathrm{to} 80$ percent of women with PCOS are obese or obese. The girls with symptoms of PCOS have hair growth on their face and framewhich include on their back, belly, and chest, referred to as Hirsutism. Acne is a symptom wherein Androgen can make the skin oilier and formation of acne on face, chest and upper back. Hormone tests includes boom in levels of LH(Luteinizing hormone), FSH(Follicle-stimulating hormone),Androgen Level, DHEAS (Dehydroepiandrosterone). Ratio of fasting insulin and fasting blood sugar if decreased, results in terrible insulin resistance.

The exact cause of PCOS is unknown. Factors that might play a role in PCOS are Excess insulin, Low- grade infection, Heredity, Excess androgen [5]. 
Insulin is a hormone that is produced in the pancreas that permits frame cells to apply sugar. Blood sugar stages upward thrust and your body might produce greater insulin. Excessive insulin may growth in production of androgen (male hormone), causing issues with ovulation. Low-grade irritation describes white blood cells'(WBC) production of substances to fight infection. Some researchers have counseled that positive genes might be linked to PCOS. The ovaries produce very excessive tiers of androgen (male hormone), resulting in hirsutism and acne.

There is no single test to diagnose PCOS. To diagnose PCOS and find out other causes of your symptoms, a doctor may talk to you about your medical history and do a physical exam and different tests like Pelvic exam, Pelvic ultrasound and some blood tests.

\section{LITERATURE SURVEY}

"Community Screening For PCOS Amongst Adolescent ladies during a Semi geographic region In West Bengal"[6], which proposed 2015 adolescent ladies of West Bengal were asked concerning their initiation of periods and about They were conjointly examined for clinical options like excess in steroid hormone. As per metropolis criteria, the cases were ended by having PCOS or not having PCOS.

"2D and 3D Trans-vaginal ultra sonography to work out Cut-offs for sex gland Volume and cyst variety per Ovary for diagnosing of Polycystic Ovary Syndrome in Indian Women" [7], which proposed eighty six girls having PCOS and forty five controls/volunteers were chosen. A 2-D and three-D trans-vaginal imaging was carried out in early menstrual days (D2 - D5). Ovary volume(OV), follicle count per ovary(FNPO), stromal volume, organic process index (VI), organic process flow index (VFI) and flow index (FI) were measured in PCOS and controls. Mann-Whitney take a look at supply regression model were accustomed to compare the info between PCOS and management.

"Two and three-dimensional sonographic and color physicist techniques for diagnosing of polycystic ovary syndrome"[8], which proposed 112 lean Italian girls having PCOS and fifty two controls /volunteers were All participants underwent transvaginal sonographic examinations (RIC5-9H, Voluson 730 knowledgeable ultrasonography system) in their vesicle part for activity of sex gland volume, cyst count, and vesicle most diameter. Continuous variables were analyzed victimization Shapiro-Wilk normality tests.

"Early Endocrine, Metabolic, and Sonographic Characteristics of Polycystic Ovary Syndrome (PCOS): Comparison between Non obese and Obese Adolescents"[9], which proposed 11 non-obese and 22 obese adolescents with PCOS and 15 obese controls were chosen. The objective was to characterize early endocrine and metabolic changes in mid-aged women with PCOS and to determine whether the differences between non-obese and obese women are detected early. The comparison between obese PCOS and non obese PCOS is done with $\mathrm{F}$ test.

"Ultrasound features of polycystic ovaries relate to degree of reproductive and metabolic disturbance in polycystic ovary syndrome"[10], which proposed 49 women (aged between 19 to 36) diagnosed with PCOS were chosen. Evaluation of menstrual cycle and also physical exam assess various parameters (height, weight, BMI, blood pressure, etc) was performed. Study of Antral follicle count(AFC), number of follicles per follicle size, ovarian volume(OV), stromal area(SA), ovarian area(OA), stromal to ovarian area(S/A), stromal index(SI) is performed. Spearman rank was used for correlation between different parameters.

"Data mining to elicit predominant factors causing infertility in women"'[11], which proposed a sample population of 575 patients who were getting treatment in endometrial research center in Trichy were selected. Physical and hormonal factors were taken into account. Based on these factors, patients were categorized into 154 fertile women and 421 infertile women. Questionnaire was created which consist of various parameters regarding the factors that influenced the infertility. Statistical analysis and CFS subset evaluator was deployed here. Two methods of classification were used J48 algorithm and Random tree algorithm. K-means clustering and Association rule mining was performed. Patients with BMI $>29$, history of diabetes, history of tuberculosis, FSH $>$ 8.5 , or $\mathrm{LH}>7$ or $\mathrm{TSH}>4$ are highly related to be infertile. $\mathrm{J} 48$ pruned tree is a relatively better technique in terms of accuracy in classifying the given record sets with an accuracy of $96 \%$. K- Means used to verify the output of previous method.

"Temperament and Character Differences of Patients with Polycystic Ovary Syndrome"[12], which proposed a total of 91 subjects were included in the study. Clinical and/or biochemical signs of hyperandrogenism; oligo/amenorrhea and polycystic ovaries in ultrasound were considered. Modified Ferriman- Gallwey score (FGS). The relationships between test scores were tested with Mann-Whitney U and independent samples t-test. PCOS patients had more problems like acne, menstrual irregularities, hirsutism, infertility and oligomenorrhea than controls $(\mathrm{p}<0.05)$. Testosteron (T) levels and the FGS, LH, follicle stimulating hormone (FSH) and BMI of the patients were not correlated significantly with depression or anxiety scores $(\mathrm{p}>0.05)$. Age and novelty seeking as well as age and reward dependence have negative correlation(r:-0.33, p:0.018; r:-0.295, p:0.037, respectively). "Coagulation parameters predictive of polycystic ovary syndrome"'[13], which proposed there were 181 patients having PCOS (selected according to Rotterdam criteria) and 301 controls were selected. Data from the coagulation assays, glucose tolerance test, and insulin release test were then collected from all study subjects. The coagulation assays included pro- thrombin time (PT), activated partial prothrombin time (APTT), thrombin time (TT), fibrinogen (FG), D-Dimer (DD), and fibrin degradation products (FDP). A linear regression model was built on the training dataset where $70 \%$ data was selected for training the model. The built linear regression was applied to the testing data for validation. The ROC curve shows AUC of 0.79. A cut point of 0.45 for the predicted probability, the sensitivity was 0.69 and the specificity 0.77 for the test dataset. Test dataset performance was similar to the performance of the training dataset. "A Classification of Polycystic Ovary Syndrome Based on Follicle Detection of Ultrasound Images"[14], which proposed 80 images, consisting of 60 normal ovary images and 20 images of PCOS ovary. Ovaries are of 2 types: normal and polycystic (multiple small cysts). Normal ovary is characterized by the count of 5-9 follicles per ovary and size 2-28 mm where as in PCOS follicles are mature enough to ovulate when their size reaches $18-28 \mathrm{~mm}$. Three classification methods were used: 
Neural Network-Learning Vector Quantization (LVQ) method

- KNN - euclidean distance

- Support Vector Machine (SVM) - RBF Kernel

"An improved Data Clustering algorithm in a Multi-objective Framework" [15], was proposed. It's about multi-objective techniques for clustering that can partition data into appropriate number of clusters. K-means is a well known algorithm for data clustering and so it was used to produce initial clusters. These clusters were further optimized using three objective functions such as compactness, connectedness and symmetry in NSGA II algorithm. The proposed hybrid clustering algorithm produces better quality clusters.

"An Improved Matching Functions for Information Retrieval Using Genetic Algorithm"[16], which proposed a novel information retrieval using genetic algorithm. This algorithm was used to increase the performance of information retrieval system. Two functions Overall Matching Function (OMF) and Virtual Center based Matching Function (VCF) helped to improve the retrieval performance. VCF based Genetic Algorithm (VCGA) are used for information retrieval. The GA steps were applied and the candidates were selected who met the threshold value. The benefit of using GA was that the queries were matched with the cluster centers and with each document.

"An efficient hybrid data clustering method based on Candidate Group Search and Genetic Algorithm" [17], in which clustering of data was performed by Candidate Group Search which calculated the initial Centroid. The Candidate Group Search reduced the time in order to perform genetic clustering. Genetic algorithm was used to assign data elements to suitable cluster. The distance from cluster center to the data points was found by CGS. The set of centers with highest accuracy, are selected using the fitness function of Genetic Algorithm.

"Lung Cancer Detection using Genetic Approach"[18], which proposed a system for early detection and diagnosis of lung cancer from X - Ray, CT and PET images. Genetic algorithm was used for optimization of results. This optimization results will help the doctors to identify the nodules present in the lungs at early stages. To improve classification performance of large scale of data, Genetic Algorithm (GA) was proposed.

"New Unification Matching Scheme for efficient information retrieval using Genetic Algorithm" [19], which proposed a new Unification Matching Scheme (UMS) for information retrieval using Genetic Algorithm. The UMS executes Unification function on three classical matching functions for different threshold values and the best results from each matching function define the new generation. The results from each generation are optimized using Genetic Algorithm. "Novel Multi Stage Genetic Clustering for Multi-objective optimization in Data Clustering" [20], which proposed a new Multi Stage Genetic Clustering (MSGC) scheme for multi-objective optimization in data clustering. MSGC can automatically partition the data into appropriate number of clusters. Genetic algorithm (GA) was applied at two stages with four objective functions. Two objective functions give the initial partitions in first stage and other two give optimal clustering centers.

\section{PROPOSED SYSTEM}

\section{A. Work Flow of Proposed Approach}

The proposed work is categorized into two phases. In phase I, machine learning algorithms are applied to classify given data as PCOS and non-PCOS. The blood test results and sonograms are the inputs for this module. In second phase, the classified data with PCOS are further optimized using Genetic Clustering approach. The results are also compared with other hybrid algorithms.

\section{Phase I: Classification of PCOS data}

The patients with metabolic (physical) symptoms like acne, facial hair growth and irregular period's can be examined in daily routine. But, these metabolic symptoms alone are not sufficient to diagnose the PCOS. Therefore the hormone tests like LH (Luteinizing hormone), FSH (Follicle-stimulating hormone), androgen level, DHEAS, fasting insulin, fasting blood sugar should be examined. The physical as well as hormonal symptoms will be considered as a feature set for the proposed system. These features will be statistically analyzed with machine learning algorithms. By using feature extraction algorithm the formation of the cysts will be studied and included in the feature set. Using appropriate classification algorithm, the prediction about the patient having PCOS will be determined. Fig. 2 shows the workflow of the proposed approach.

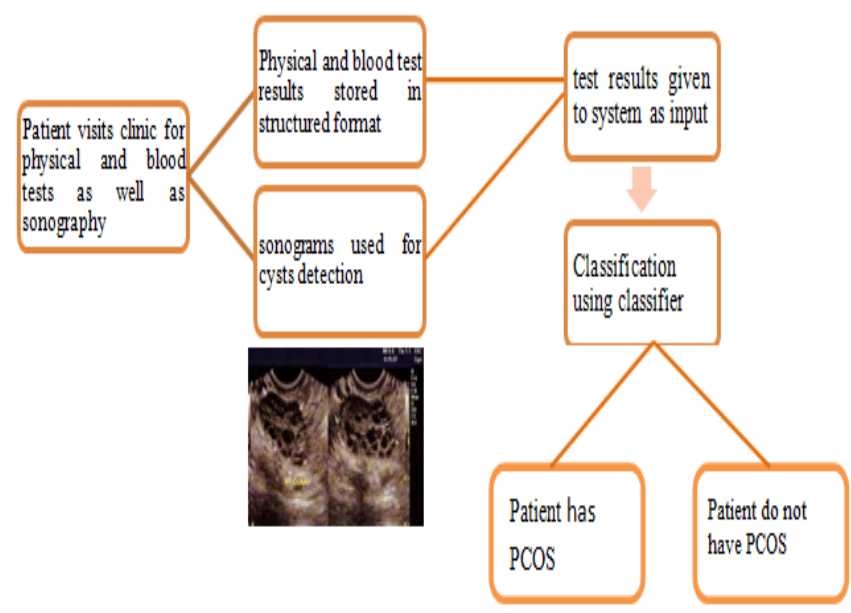

Fig. 2 Phase I: Work flow of classification of PCOS

\section{Phase II: Optimization with Genetic Clustering}

The classified instances as PCOS are further optimized using Genetic Algorithm. In this phase the fitness of every instance is calculated based on its weighted output of all parameters to correctly detect PCOS. All the fitness values are then ranked to understand the capability of every instance. With the threshold of 40 percent, the highest fitness instances are directly considered for next generation whereas; remaining instances are exposed to various genetic operators. The operators like selection, crossover and mutation are applied on these instances to check new target regions. The input data instances are encoded with real numbers between 0 to 1 using normalization techniques. Stochastic universal sampling method is used for selection the parent chromosomes. 
For crossover operation, single crossover strategy is used with 0.8 percent crossover rate. The mutation rate of 0.1 percent is applied. During experimentation, the results for various generations are computed and compared. The convergence point is recorded and final accuracy parameters are recorded. Fig. 3 shows the genetic clustering functioning.

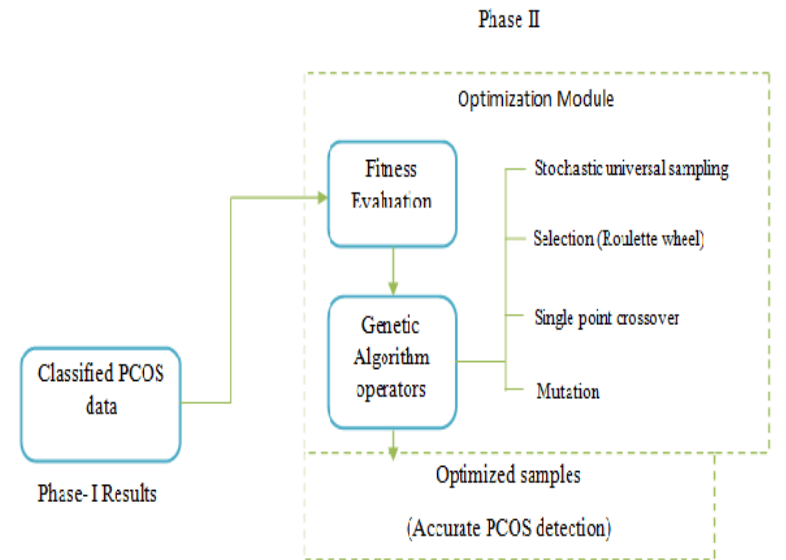

Fig. 3 Phase II: Genetic clustering for PCOS predicted data

\section{RESULTS AND DISCUSSION}

\section{A. Datasets:}

The dataset for proposed system is not readily found on available repositories. Therefore, dataset is created in discussion with medical practitioner with their expertise in PCOS detection. Figure 4 represents a small snippet of the dataset created. The dataset generated has 13 attributes and 2 classes. Total 84 instances are created. These attributes are the symptoms related to PCOS such as physical symptoms(age, height, weight, irregular periods, hirsutism, acne), and blood test results; (LH(Luteinizing hormone), FSH(Follicle-stimulating hormone), androgen level, DHEAS, fasting insulin, fasting blood sugar) and clinical test (sonography). The class type specifies the presence of PCOS or not. The most important symptoms (that are influencing factors) as highlighted are weight, irregular periods, acne, LH and sonography. The type attribute tells about the prediction whether the women has PCOS or not. The dataset is validated based on various cases of PCOS patients and opinion of expert from medical domain. Fig. 4 shows a sample of dataset.

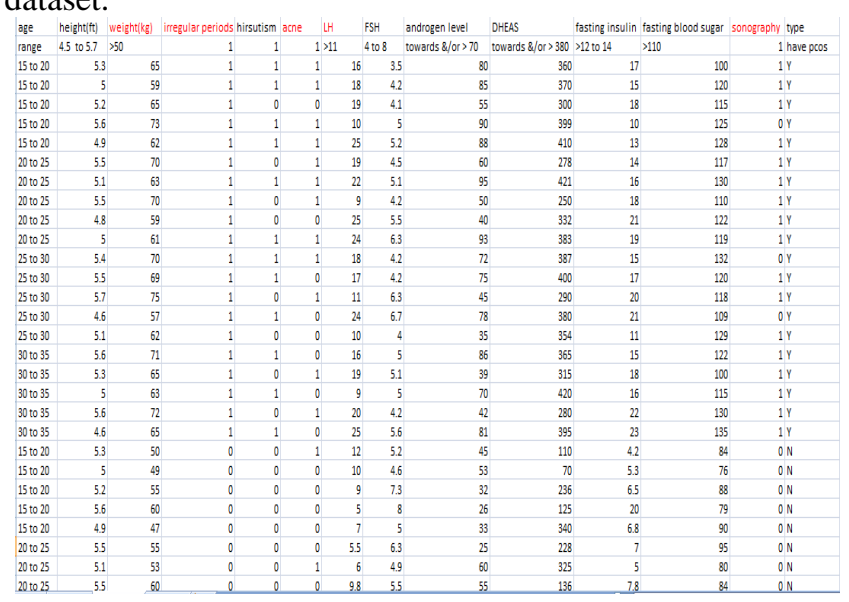

Fig. 4 Sample dataset

\section{B. Performance Measures:}

I. Accuracy:

Accuracy is the most sensitive performance measure. It is simply a ratio of correct predicted observations to total number of observations. Accuracy is a great measure only when the values of false positive and false negative are almost same.

$$
\text { Accuracy }_{P \cos }=\frac{T P+T N}{T P+F P+F N+T N}
$$

\section{Precision:}

Precision is a ratio of correctly predicted positive observations to the total predicted positive observations.

$$
\text { precision }_{P C O S}=\frac{T P}{T P+F P}
$$

III. Recall:

Recall is a ratio of correctly predicted positive observations to all observations in actual class.

recall $_{P C O S}=\frac{T P}{T P+F N}$

IV. F-measure:

$\mathrm{F}$ - measure score takes both false positive and false negative into consideration. It is a weighted average of Precision and Recall. It is more useful than Accuracy.

$$
F-\text { measure }_{\text {PCOS }}=\frac{2 *(\text { recall } * \text { precision })}{(\text { recall }+ \text { precision })}
$$

\section{Experimentation and Discussion}

The experimentation is carried out on the dataset created and well known machine learning algorithms. The objective of using various algorithms is to identify the most suitable algorithms for classification of the dataset created.

Phase I Implementation:

The Machine Learning algorithms like Artificial Neural Network, K-nearest Neighbor and Linear Regression are used for classification and performance is analyzed statistically. Statistical result like Accuracy is calculated and compared using functions available in Python. Table I depicts the statistical results.

Table I Performance measures of various classification algorithms

\begin{tabular}{|l|c|c|c|}
\hline Algorithms & $\begin{array}{l}\text { Artificial } \\
\text { Neural } \\
\text { Network }\end{array}$ & $\begin{array}{l}\text { K-nearest } \\
\text { neighbor }\end{array}$ & $\begin{array}{l}\text { Linear } \\
\text { Regression }\end{array}$ \\
\hline Accuracy & 0.94 & 0.5 & 1 \\
\hline Precision & 0.89 & 0.5 & 1 \\
\hline Recall & 1 & 1 & 1 \\
\hline F - measure & 0.94 & 0.67 & 1 \\
\hline
\end{tabular}




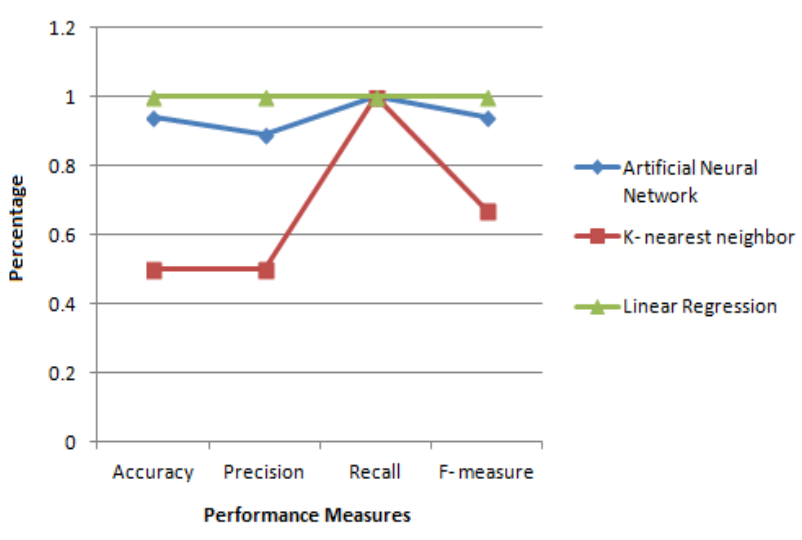

Fig. 5 Performance measures of PCOS with various machine learning algorithms

From the results shown in Fig. 5, it is observed that in all statistical parameters, Linear Regression algorithm super sits the other algorithms, giving good classification accuracy. Whereas; other algorithms may outperform with increased dataset size. This analysis will be carried out further for the said research.

Phase II Implementation:

The results of Phase I, are found to be good for Linear regression. Therefore, in this phase the results of linear regression are optimized using Genetic Clustering. Table II depicts the results of Genetic Clustering. The initial parameters for phase II to calculated optimal set from classified instances of PCOs are;

PCOS classified instances (No. of samples): $N$

Crossover Rate: $C_{R}=0.8$

Mutation Rate: $M_{r}=0.1$

Fitness Function: $F_{n}$ (Intracluster distance)

Number of generations: $G_{n}=300$

Table II The results of Genetic Clustering.

\begin{tabular}{|c|c|c|c|}
\hline $\begin{array}{c}\text { No. of } \\
\text { generations }\end{array}$ & $\begin{array}{c}\text { Centroid } \\
\text { value }\end{array}$ & Threshold & $\begin{array}{c}\text { Accuracy } \\
\text { (\%) }\end{array}$ \\
\hline 50 & 0.081 & $60 \%$ & 79 \\
\hline 100 & 0.077 & $60 \%$ & 81 \\
\hline 200 & 0.072 & $60 \%$ & 88 \\
\hline 300 & 0.069 & $60 \%$ & 89 \\
\hline
\end{tabular}

The results are computed considering various generations. The Centroid values are the values of final generation. These centroids will form final clusters. Threshold is nothing but the percentage of instances considered as fittest individuals after ranking for next generation. Accuracy is calculated based on number of instances correctly placed in cluster i.e. with minimum error rate.

\section{CONCLUSION}

The classification of PCOS using machine learning algorithms i.e. Artificial Neural Network using relu activation, K-Nearest algorithm and Linear Regression algorithm are experimented. The result helps in prediction of whether the patient has Polycystic Ovarian Syndrome or not. These results are based on readings of hormonal imbalance, physical symptoms and sonograms. It is observed that Linear Regression gave highest accuracy of classification. In the second phase of implementation, the classified instances of PCOS are further optimized using Genetic Clustering. The classification results are optimized with $89 \%$ accuracy and reduced error rate.

\section{REFERENCES}

1. https://www.healthline.com/health/polycystic-ovary-disease

2. https://www.healthline.com/health/polycystic-ovary-disease\#what-ispcos

3. Uche Anadu Ndefo, Angie Eaton, and Monica Robinson Green, "Polycystic Ovary Syndrome, A Review of Treatment Options With a Focus on Pharmacological Approaches", P T. 2013 Jun; 38(6): 336-338, 348, 355

4. https;//www.mayoclinic.org/diseases-conditions/pcos/symptoms -causes/syc-20353439

5. https://www.medicalnewstoday.com/articles/265309.php\#symptoms

6. Jayanta Pal, Barindra Nath Mallick, "Community Screening For PCOS Amongst Adolescent Girls In A Semiurban Area In West Bengal", J. Evid. Based Med. Healthc., pISSN 2349-2562,eISSN2349-2570/ Vol. 3/Issue 100/Dec. 15, 2016

7. Kar Sujata , Samparna Swoyam, "2D and 3D Trans-vaginal Sonography to Determine Cut-offs for Ovarian Volume and Follicle Number per Ovary for Diagnosis of Polycystic Ovary Syndrome in Indian Women", J Reprod Infertil. 2018;19(3):146-151

8. Cesare Battaglia, Bruno Battaglia, Elena Morotti, Roberto Paradisi, Isabella Zanetti, Maria Cristina Meriggiola, Stefano Venturoli, "Twoand Three-Dimensional Sonographic and Color Doppler Techniques for Diagnosis of Polycystic Ovary Syndrome", J Ultrasound Med 2012; 31:1015-1024

9. Miriam e. Silfen, michelle r. Denburg, alexandra m. Manibo, rogerio a. Lobo, Richard jaffe, michel ferin, lenore s. Levine, and sharon e. Oberfield, "Early Endocrine, Metabolic, and Sonographic Characteristics of Polycystic Ovary Syndrome (PCOS): Comparison between Nonobese and Obese Adolescents", JCEM88(10):4682-4688 Copyright (C) 2003 by The Endocrine Society. doi: 10.1210/jc.2003-030617

10. Jacob P. Christ, Heidi Vanden Brink, Eric D. Brooks, Roger A. Pierson, Donna R. Chizen and Marla E. "Ultrasound features of polycystic ovaries relate to degree of reproductive and metabolic disturbance in polycystic ovary syndrome", Lujan Fertility and Sterility. Vol. No. 2015 0015- 0282/\$36.00

11. Ms. N. Vijayalakshmi, Ms. M. UmaMaheswari, "Data mining to elicit predominant factors causing infertility in women ", IJCSMC, Vol. 5 Issue. 8, August 2016

12. Ahmet O" ztu“ rk, Suna Kabil Kucur, Ali Seven, Erdem Deveci, Halime S, encan, Alperen Kilic, Onur Yilmaz, Alperen Kilic, "Temperament and Character Differences of Patients with Polycystic Ovary Syndrome", PII: S2468-7847(18)30314-3 DOI:https://doi.org/10.1016/j.jogoh.2019.01.006Reference: JOGOH 1527, 22 January 2019

13. Qian Suna, Yan Yangb, Xuenan Pengc, Yunyan Zhangb, Yuan Gaoa, Fang Wanga, Yang Zhanga, Wen Fenga, Wen Yanga, Xiaomin Kang, "Coagulation parameters predictive of polycystic ovary syndrome", https://doi.org/10.1016/j.ejogrb.2019.06.018 0301-2115/C 2019 Elsevier B.V. All rights reserved, Accepted 12 June 2019

14. Bedy Purnama, Untari Novia Wisesti, Adiwijaya, Fhira Nhita, Andini Gayatri, Titik Mutiah, "A Classification of Polycystic Ovary Syndrome Based on Follicle Detection of Ultrasound Images", 978-1-4799-7752-9/15/\$31.00 @2015 IEEE

15. Anuradha D. Thakare, M.A. More, "An improved Data Clustering algorithm in a Multiobjective Framework", 2014 Annual IEEE India Conference (INDICON)

16. Prof. Anuradha D. Thakare, Dr. C.A. Dhote, "An Improved Matching Functions for Information Retrieval Using Genetic Algorithm", 978-1-4673-6217-7/13/\$31.00_c 2013 IEEE

17. Suvarna P. Patil, Anuradha D. Thakare, C. A. Dhote, "An efficient hybrid data clustering method based on Candidate Group Search and Genetic Algorithm", 978-1-4673-7231-2/15/\$31.00 (C2015 IEEE

18. Manasee Kurkure, Anuradha Thakare, "Lung Cancer Detection using Genetic Approach", Computing Communication Control and automation (ICCUBEA), 2016 International Conference on, Publication in IEEE Digital Library : 23 February 2017, Pages: 1-5, ISBN: 978-1-5090-3291-4

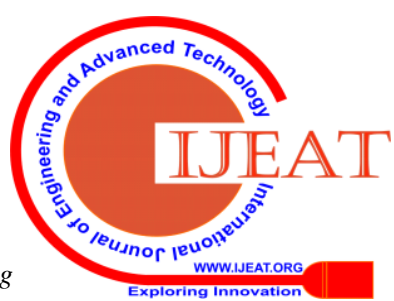


19. Anuradha D. Thakare, C.A. Dhote, "New Unification Matching Scheme for efficient information retrieval using Genetic Algorithm", 978-1-4799-3080-7/14/\$31.00_c 2014 IEEE

20. Anuradha D. Thakare, C.A. Dhote, "Novel Multi Stage Genetic Clustering for Multiobjective optimization in Data Clustering", 2015 International Conference on Computing Communication Control and Automation

\section{AUTHORS PROFILE}

Dr. Anuradha Thakare, (Doctorate from SGB Amravati University) is a Professor in Computer Engineering in Pimpri chinchwad college of Engineering of Savitribai Phule Pune University. Her area of research is Machine Learning, Data Analytics and Artificial Intelligence. She has written various research articles and papers which are published in Journals and conferences of high repute. She received grant from BCUD, SPPU, MSCW, QIP Program SPPU and AICTE for research projects and research activities. Dr. Anuradha also worked for consultancy projects and research projects with industries. Currently, she is working in Cognitive Domain and IT Enabled Healthcare projects. Dr. Anuradha is representing IET (Institution of Engineering \& Technology) Pune LN as a Secretary.

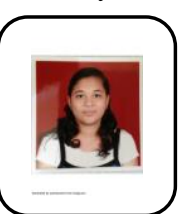

Priyanka R Lele, is a post- graduate student in Computer Engineering in Pimpri Chinchwad College of Engineering at Savitribai Phule Pune University. Her area of research is Machine Learning, Data Mining, Data Analytics and Image Processing. For her ongoing project she has asked for the doctor's expertise in her research. Her Bachelor project was in data mining domain. The project was about online admission for non - centralized courses for all three fields - arts, commerce and science. Currently, she is working on image processing for feature extraction of the sonography results. Also she is learning about some deep learning concepts to further apply it on the project. 